\title{
Local Density of States and Level Width for Wannier-Stark Ladders
}

\author{
M.C.Chang and Q.Niu \\ Department of Physics, University of Texas, Austin,TX 78712
}

\begin{abstract}
The local density of states $\rho(x, E)$ is calculated for a Bloch electron in an electric field. Depending on the system size, we can see one or more sequences of Wannier-Stark ladders in $\rho(x, E)$, with Lorentz type level widths and apparent spatial localization of the states. Our model is a chain of delta function potential barriers plus a staircase-like electric potential, with open boundary condition at both ends of the system. Using a wave tunneling picture, we find that the level widths shrink to zero as an inverse power as the system size approaches infinity, confirming an earlier result. The level width will not approach zero if the delta function barriers are replaced by the Kronig-Penny potential or smoother ones, as is commonly believed.
\end{abstract}

PACS: 71.20.-b, 71.50.+t, 73.20.Dx, 73.40.Gk

To be published in Phys. Rev.B47, July (or August), 1993.

changmc@utpapa.ph.utexas.edu 


\section{Introduction}

It is well known that an electron in an isolated Bloch band will oscillate with frequency $e F a / h$ in the Brillouin zone when it is subject to a uniform and constant electric field $F$, where $a$ is the lattice constant. It was first pointed out by Wannier that such oscillatory motion is associated with a ladder like spectrum, now commonly known as Wannier-Stark ladders. Wannier further proposed that sequences of such ladders exist even when the interband coupling is taken into account.[1]

This proposal was immediately challenged by Zak, who pointed out that sharp WannierStark ladders cannot exist in a system with any reasonably continuous periodic potential, because the spectrum is absolutely continuous.[2] A numerical study on the density of states $\rho(E)$ for a system with a cosine potential in an electric field also showed no evidence of the ladder structure [3]. Since the existence of sharp Wannier-Stark ladders in the one band approximation was rigorously established [4], it was then suggested that a correct account of the interband transitions will smear out the discrete spectrum [5]. Further, it has also been proved that an isolated group of $n$ Bloch bands can give rise to $n$ sequences of sharp ladders, so one must consider an infinite number of coupled bands in order to obtain a continuous spectrum. Recently, by decomposing the linear electric potential into a sawtooth part plus a staircase part, Emin and Hart claimed that the bands may be effectively decoupled because the interband matrix elements of the staircase potential is zero. This would give a proof for the existence of a sharp ladder spectrum, but the arguments leading to the vanishing of interband matrix elements have been critcized[6].

On the other hand, experimental and theoretical evidence in support of the existence of Wannier-Stark ladders also increased during the same period of time [7]. Krieger and Iafrate clarified the controversies concerning the use of periodic boundary condition and the neglect of interband transitions.[8] Although they did not discuss the energy spectrum at all, they can show that the transitions of electrons induced by an incident electromagnetic field are allowed only if $\Delta E=e F a$, as if these levels exist. In recent years, thanks to technological advancement on superlattices, the existence of a ladder structure seems to be firmly established. [9] 
A clear picture about the nature of the W-S ladders should have emerged from the many years of contraversial debates: the ladders are not infinitely sharp levels, but they are resonant levels whose life times can be long under conditions of small interband couplings. In earlier numerical works, such resonant levels are located by searching for poles of the S matrix or the resolvant in the complex energy plane, with the level widths given by the imaginary part of the poles.[7] In this paper, we reveal and display the W-S ladder structure in the local density of states $\rho(x, E)$, which can be calculated in a simple and direct way. As we will show in the main text, the W-S ladders appear naturally and very clearly in a plot of $\rho(x, E)$ as sequences of mountains, whose widths in the $E$ direction represent the level widths, and whose widths in the $x$ direction represent the localization of the ladder states. Such structures can be easily washed out in the total density of states $\rho(E)$.

In order to simplify the calculation, we use in our model a chain of delta function barriers to simulate the periodic potential, and use a staircase potential to simulate the external field. There has been criticism concerning the use of the staircase potential, arguing that it will lead to vanishing interband transitions [10], however, such criticism is invalid as has been shown by Kleinman[6] and others. Although our model is used mainly for qualitative studies, it can also be used to simulate real solids when the spatial extent of the wave functions are large compared to the lattice constant as has been demonstarted by Bentosela et al in a similar situation.[7]

The organization of this paper is as follows: In Sec.2 we describe our model and derive a formula for the local density of states. In Sec.3 we present the numerical results for the local and total density of states with a variety of parameter values. In Sec.4, the behavior of the level width as a function of system size and other parameters is explained in a picture of wave tunneling through the potential barriers.

II. The Model 
We will study the wave function solution of a one dimensional model with Hamiltonian

$$
H=-\frac{\hbar^{2}}{2 m} \frac{d^{2}}{d x^{2}}+V_{0} a \sum_{j=-N_{l}}^{N_{r}} \delta(x-j a)+V_{e x t}
$$

where $V_{e x t}$ is a staircase potential, simulating an external electric field. Reference of energy is chosen such that

$$
V_{\text {ext }}=-j \Delta \text { for } j a<x<(j+1) a, j \in Z
$$

We will take $\hbar^{2} \pi^{2} / 2 m a^{2}$ as the unit of energy, therefore $\Delta=0.2$ corresponds to an electric field of $F=7 \times 10^{4}(\mathrm{~V} / \mathrm{cm})$ for $a=200 \AA$ and $m=0.067 m_{e}$.

We will choose our boundary such that the potential beyond both ends of our crystal levels off, as shown in Fig.1. The cells are numbered by the integer $j$ in (2). The wave function in cell $j$ is

$$
\psi_{j}(x)=a_{j} e^{i k_{j} x}+b_{j} e^{-i k_{j} x}
$$

where $k_{j}=(\pi / a) \sqrt{E+j \Delta}$ (new unit). The coefficients of cell $j+1$ and cell $j$ are related by a transfer matrix $\mathbf{M}_{\mathbf{j}}$ :

$$
\left(\begin{array}{c}
a_{j+1} \\
b_{j+1}
\end{array}\right)=\mathbf{M}_{j}\left(\begin{array}{c}
a_{j} \\
b_{j}
\end{array}\right)
$$

where

$$
\mathbf{M}_{j}=\frac{1}{2 k_{j+1}}\left(\begin{array}{cc}
\left(k_{j}+k_{j+1}-i \kappa\right) e^{-i\left(k_{j+1}-k_{j}\right)(j+1) a} & \left(-k_{j}+k_{j+1}-i \kappa\right) e^{-i\left(k_{j+1}+k_{j}\right)(j+1) a} \\
\left(-k_{j}+k_{j+1}+i \kappa\right) e^{i\left(k_{j+1}+k_{j}\right)(j+1) a} & \left(k_{j}+k_{j+1}+i \kappa\right) e^{i\left(k_{j+1}-k_{j}\right)(j+1) a}
\end{array}\right)
$$

and $\kappa \equiv \pi^{2} V_{0} / a$. The above formula remains valid when $E<-j \Delta$, in which case we take $k_{j}=-i(\pi / a) \sqrt{|j \Delta+E|}$. Notice that $\operatorname{det} \mathbf{M}_{j}=k_{j} / k_{j+1}$, which is not equal to the usual value of unity, because the velocities corresponding to a given energy in neighboring cells are different.

In our study we will take $E<N_{l} \Delta$, and drop off the exponentially large solution at the left edge by letting $b_{-N_{l}-1}=0$. The two solutions $a_{N_{r}} e^{i k_{N_{r}} x}, b_{N_{r}} e^{-i k_{N_{r}} x}$ beyond the right edge correspond to incoming and outgoing waves. The normalization of wavefunctions is set by taking $\left|a_{N_{r}}\right|=1$. Furthermore, in the actual calculation, the number of cells on the 
negative $x$ axis is limited to 10 for convenience. The error involved is negligible as long as we are considering energy only a few $\Delta$ 's large.

The quantity we are after is the local density of states defined by

$$
\begin{aligned}
\rho(x, E) & =\int_{0}^{\infty} \frac{d k}{2 \pi}\left|\psi_{k}(x)\right|^{2} \delta\left(E-E_{k}\right) \\
& =\frac{1}{2 \pi \hbar} \sqrt{\frac{m}{2\left(E+N_{r} \Delta\right)}}\left|\psi_{k}(x)\right|_{E_{k}=E+N_{r} \Delta}^{2}
\end{aligned}
$$

where $E_{k}=(k a / \pi)^{2}$ Unlike the total density of states $\rho(E)=\int d x \rho(x, E)$ which is usually calculated, the local density of states also contains information about the location of states, and is more appropriate for the purpose of revealing the nature of the W-S ladders.

\section{Result}

The coefficients $a_{j}, b_{j}\left(j=-\left(N_{l}+1\right) \sim N_{r}\right)$ are calculated starting from $b_{-N_{l}-1}=0$, and $a_{-N_{l}-1}$ is chosen such that $\left|a_{N_{r}}\right|=1$. We can get $\rho(x, E)$ for some fixed value of energy $E$. The energy is then changed to a different value and the calculation is done all over again. The result is double checked by calculating $a_{j}, b_{j}$ 's starting from $a_{N_{r}}$ and $b_{N_{r}}$.

When the electric field is zero, we should see an energy band structure, as shown in Fig.2. There are 50 cells $\left(N_{l}=10, N_{r}=40\right)$ in this system. Only the first band and the lower half of the second band are plotted. The band edges coincides very well with a simple theoretical calculation.

Fig.3 is a plot of $\rho(x, E)$ after we turn on the electric field. The lattice is composed of 50 cells ( labelled $-10 \sim 39$ ). Only 12 cells $(-1 \sim 10)$ are plotted here. The energy range covered is $3 \Delta$ 's wide. Two sequences of states can be seen. The first sequence lies at energies equal to $(0.34+n) \Delta$, where $n=$ integer and is equal to 0,1 and 2 in the energy range of the figure. The ladder at $E=0.34 \Delta$ has a primary peak in cell 1 and 2 , with smaller peaks in the cells to the right. The second sequence is weaker and broader, but is still discernible, and lies at energies equal to $(0.65+n) \Delta$ with $n=0,1,2$ in the figure. The ladder at $E=0.65 \Delta$ is primarily peaked in cell 8 , with the heights of the peaks slowly decreasing to the left and right. It is evident that $\rho(x, E)$ is invariant under changes $x \rightarrow x+a, E \rightarrow E-\Delta$ as it should be. Each state in both sequences spans many 
lattice sites, and will not 'feel' much difference whether the electric potential is linear or staircase-like.

The total density of states $\rho(E)$ is obtained by integrating $\rho(x, E)$ with respect to $x$ up to the right edge of the lattice. The result is shown in Fig. 4 (a) (note the difference from the result in the absence of the field shown in Fig. 2). The two peaks within each $\Delta$ in energy correspond to the two sequences that can be observed in the local density of states. As we enlarge the system, the number of peaks increases (see Fig. 4 (b),(c),(d) ), corresponding to the increased number of observable sequences. One can imagine that as the system becomes infinite, there will be infinite number of peaks covering up the whole energy axis, making the ladder structure unrecognizable in $\rho(E)$. Fig. 5 is a plot for an electric field that is five times larger than in Fig.4, with more peaks showing up in $\rho(E)$ corresponding to more sequences of observable ladders in the system.

For a system with $\left(V, \Delta, N_{r}\right)=(1,0.2,40)$, two sequences are observed, as in Fig.3, but with much narrower level widths. In Fig.6, the spatial extent of the states in these two sequences are plotted. One is at $E \simeq 0.3 \Delta$, the other is at $E \simeq 0.7 \Delta$. The state in Fig.6(b) is essentially confined within $3 \sim 5$ cells, which is more localized than the states in the first 'band' in Fig.3. A cross section of it at $x=3.5 a$ is plotted in Fig.7. It is so sharp that energy resolution as high as $\Delta / 10^{11}$ has to be used. A nice Lorentz curve appears after such an immense blow up. The width of the state in Fig.6(a) is about $10^{-6} \Delta$.

Fig. 8 is for another set up: the lattice has 14 cells $(-10 \sim 3)$, with a higher barrier $V_{0}$. It is a cross section of the lowest peak of cell 0 . This peak is essentially confined within one cell. It is almost as sharp as the peak in Fig. 7(b) even though we are considering a relatively small system - there are only 4 cells on the positive $x$-axis, because the barriers are much higher. Its width will be calculated by wave tunneling in Sec. 4 .

The level width will decrease as $N$ increases, because it is more difficult for these resonant states to tunnel out. An interesting question is: when our system becomes macroscopically large, will the width shrink to zero or to a finite value? This is answered in the next section.

IV. Tunneling and level width 


\section{A. General formula}

We will be interested in those resonant levels mainly located in a unit cell, say the lowest level in cell 0 . In the limit of small level width, we may write the level width as

$$
\delta E=(\hbar / 2)\left|T_{0}\right|^{2} \nu
$$

where $\nu$ is the attempting frequency in cell 0 , and $T_{0}$ is the transmission coefficient from cell 0 to the right edge of the system. It is emphasized that the transmission coefficient used here and hereafter refers to the probability currents instead of the probability amplitudes. The transmission coefficient through the $j$ th barrier is the same for both directions, and is given by

$$
t_{j}=\frac{2 i \sqrt{k_{j-1} k_{j}}}{i\left(k_{j-1}+k_{j}\right)-\kappa} e^{i\left(k_{j-1}-k_{j}\right) a j}
$$

On the other hand, the reflection coefficient of a barrier depends on the direction of incidence, and is given by

$$
\begin{aligned}
& r_{j \vdash}=\frac{i\left(k_{j}-k_{j-1}\right)+\kappa}{i\left(k_{j}+k_{j-1}\right)-\kappa} e^{-2 i k_{j} a j} \\
& r_{j \dashv}=\frac{i\left(k_{j-1}-k_{j}\right)+\kappa}{i\left(k_{j-1}+k_{j}\right)-\kappa} e^{2 i k_{j-1} a j}
\end{aligned}
$$

where $\vdash$ refers to reflection from and to the right, and $\dashv$ from and to the left.

A general formula for $T_{0}$ may be obtained through a diagrammatic analysis. We denote $T_{j}$ to be the transmission coefficient from the $j$ th cell to the right edge, with the understanding that all the paths are contained between the $j$ th cell and the right edge. It is then easy to show that

$$
\begin{aligned}
T_{0} & =t_{1} T_{1}+t_{1} \Sigma_{1} T_{1}+t_{1} \Sigma_{1}^{2} T_{1}+\ldots \\
& =\frac{t_{1}}{1-\Sigma_{1}} T_{1}
\end{aligned}
$$

where $\Sigma_{1}$ is the sum over contributions of 'self-energy' loops each of which goes from cell 1 to the right and resumes the initial position and direction of propagation only in the final step. See Fig. 9 (a).

The above formula can be iterated, yielding

$$
T_{0}=\prod_{j=1}^{N_{r}} \frac{t_{j}}{1-\Sigma_{j}}
$$


where $\Sigma_{j}$ is the total contribution of 'self-energy' loops starting and ending in cell $j$. It is understood that all the paths for $\Sigma_{j}$ are on the right of cell $j$, except for the first and the last steps which are in the $j$ th cell.

The formula (11) is an exact result, but the 'self-energy' contribution cannot be obtained exactly. In the case of strong barriers, we may approximate $\Sigma_{j}$ by $r_{j \vdash} r_{j+1 \dashv}$, coming from a path reflected once by the $(j+1)$ th barrier and once by the $j$ th barrier. All the other paths must have at least two transmissions through the $(j+1)$ th barrier and are therefore of higher order. We have done a calculation for the case of $N_{r}=4, \Delta=0.2$, and $V_{0}=8$. The half width is found to be $6.64 \times 10^{-10}$ by the above approximation, while the exact numerical result as shown in Fig. 8 is $(6.4 \pm 0.1) \times 10^{-10}$. The error is less than $5 \%$.

When the barriers to the right of the $j$ th cell are not strong, we cannot use the above approximation. However, if the reflection coefficients are small, we only need to sum over those loops containing 2 reflections ( Fig. 9 (b) ). Therefore,

$$
\Sigma_{j} \simeq \sigma_{j}^{(2)} \equiv \sum_{i(>j)}^{N_{r}} t_{j i} r_{i \dashv} t_{i j} r_{j \vdash}
$$

where $t_{j i}=t_{j+1} \cdots t_{i-1}$ for $j<i, t_{1 j}=t_{1} \cdots t_{j}, t_{j N}=t_{j+1} \cdots t_{N}$.

$$
T \simeq T^{\prime}=\prod_{j=1}^{N_{r}} \frac{t_{j}}{1-\sigma_{j}^{(2)}}
$$

It is interesting to note that the above formula is even valid in the opposite limit of small $t$ 's, because then only the $i=j+1$ term survives. This result is identical to the approximate formula that we derived before for the strong barrier case.

B. $\delta$-function barriers.

We are now ready to calculate the energy level width of a $\delta$ potential chain subjected to electric field. When $k_{j} \gg \kappa$, which is true for a large $j$ such that $j \Delta+E \gg V_{0}^{2}$,

$$
\begin{gathered}
\left|t_{j}\right| \simeq \frac{1}{\sqrt{1+\left(\pi V_{0} / 2\right)^{2}(1 /(E+j \Delta))}} \\
\left|r_{j \vdash}\right| \simeq\left|r_{j \dashv}\right| \simeq \frac{\pi V_{0}}{2} \frac{1}{\sqrt{E+j \Delta}}
\end{gathered}
$$


If we are considering the limit $\Delta \rightarrow 0,(14),(15)$ remain valid if $E \gg V_{0}^{2}$.

Using these approximations, we will find that : (phase factors are included)

$$
\sigma_{j}^{(2)} \simeq-\frac{\alpha}{\Delta}(j+E / \Delta)^{\beta-1}\left(\sum_{l=j}^{N_{r}} \frac{1}{(l+E / \Delta)^{\beta}} e^{i \xi l^{3 / 2}}\right) e^{-i \xi j^{3 / 2}}
$$

where $\xi \equiv(4 \pi / 3) \sqrt{\Delta}, \beta \equiv 1 / 2+\alpha / \Delta, \alpha \equiv\left(\pi V_{0} / 2\right)^{2}$. This expression is correct as long as $N \Delta$ is larger than, or of the same order of magnitude as $E$.

The problem now is how to sum up this series? If $\Delta$ is not too small, the difference $\delta\left(\xi l^{3 / 2}\right) \simeq(3 / 2) \xi l^{1 / 2}=2 \pi \sqrt{l \Delta} \gg 2 \pi$. In this case $\xi l^{3 / 2} \bmod 2 \pi$ can be considered random. Therefore, $\left(\sigma_{j}^{(2)}\right.$ is simply written as $\sigma_{j}$, and $N_{r}$ is written as $N$ in the following equations )

$$
\left|\sigma_{j}\right| \simeq \sqrt{\left\langle\left|\sigma_{j}\right|^{2}\right\rangle} \simeq \sqrt{\frac{\alpha}{2 \Delta}}(j+E / \Delta)^{-1 / 2}
$$

where \langle\rangle means taking the average with respect to the angle of the complex number. We assumed $N$ is so large that the term $\left(N+\frac{E}{\Delta}\right)^{-1 / 2}$ is negligible.

The quantity $\left|\sigma_{j}\right|$ is small since we are considering $j>J$. The infinite product $\Pi\left(1-\sigma_{j}\right)^{-1}$ in (13) can then be approximated by $e^{\sum_{j} \sigma_{j}}$ when the index $j$ is larger than $J$. The phase of $\sigma_{j}$ is, again, a random number. Therefore, we get

$$
\left.\left|\sum_{j>J}^{N} \sigma_{j}\right| \simeq \sqrt{\left\langle\left|\sum_{j>J}^{N} \sigma_{j}\right|\right.}\right\rangle \sqrt{\frac{\alpha}{2 \Delta}}\left(\ln \left(\frac{N \Delta+E}{J \Delta+E}\right)\right)^{1 / 2}
$$

with a random phase factor $e^{i \theta}$.

Finally, we get

$$
\begin{aligned}
\left|T^{\prime}\right| & =C_{J}\left|\prod_{j>J}^{N} t_{j} \prod_{j>J}^{N} \frac{1}{1-\sigma_{j}}\right| \\
& \simeq C_{J} \exp \left(\sum_{j>J}^{N} \ln \left|t_{j}\right|\right)\left|\exp \left(\sum_{j>J}^{N} \sigma_{j}\right)\right| \\
& \simeq C_{J} \exp \left(-\frac{\alpha}{2 \Delta} \ln \left(\frac{N \Delta+E}{J \Delta+E}\right)+\sqrt{\left.\frac{\alpha}{2 \Delta} \ln \left(\frac{N \Delta+E}{J \Delta+E}\right) \cos \left(\theta_{r n d}\right)\right)}\right.
\end{aligned}
$$

in which $C_{J}$ is a finite number. We can see that $T^{\prime}$ will approach zero as $N \rightarrow \infty$ 
$T^{\prime}$ is a good approximation to correct $T$, at least up to the first order of approximation. It is improbable that higher order corrections will lead to a nonzero $T$ since that would require the correction of the part of $\prod\left(1-\sigma_{j}\right)^{-1}$ diverges at least as fast as $\exp ((\alpha / 2 \Delta) \ln (N / J))$. Notice that the dominant term $-(\alpha / 2 \Delta) \ln \left(\frac{N \Delta+E}{J \Delta+E}\right)$ comes solely from the zeroth order contribution. The wavefunction $\psi(x) \sim N^{-\frac{\alpha}{2 \Delta}}$ is power law localized, which is a marginal case between extended and exponentially localized cases. Similar studies of localization in the energy space also showed marginal behavior [11].

\section{The Kronig-Penny type barriers}

The same principle can be applied to the Kronig-Penny type barriers. Assume that the width and height of the barriers are $a_{1}$ and $V_{0}$ respectively, that the distance between barriers is $a_{2}$, that $a=a_{1}+a_{2}$ is the size of unit cell, and that the potential drops one $\Delta$ over distance $a$. Then, a straightforward calculation shows that, when $j$ is much larger than one

$$
\left|t_{j}\right| \simeq\left|\frac{4 k_{j}^{\prime} / k_{j}}{\left(1+k_{j}^{\prime} / k_{j}\right)^{2}-\left(1-k_{j}^{\prime} / k_{j}\right)^{2} e^{2 i k_{j} a_{1}}}\right|
$$

where $\left.k_{j}^{\prime}=\sqrt{2 m(E+j \Delta)} / \hbar ; k_{j}=\sqrt{2 m\left(E+j \Delta-V_{0}\right.}\right) / \hbar$.

When $E+j \Delta \gg V_{0}$,

$$
\frac{k_{j}^{\prime}}{k_{j}} \simeq 1+\frac{1}{2} \frac{V_{0}}{E+j \Delta}
$$

Equation (20) can be further simplified :

$$
\left|t_{j}\right| \simeq 1-\left(\frac{V_{0}}{4(E+j \Delta)}\right)^{2}\left(1-\cos \left(2 k_{j} a_{1}\right)\right)
$$

Therefore,

$$
\prod_{j=1}^{\infty}\left|t_{j}\right| \simeq\left(\prod_{j=1}^{J-1}\left|t_{j}\right|\right) \exp \left\{-\left(\frac{V_{0}}{4}\right)^{2} \sum_{j=J}^{\infty} \frac{1}{(j \Delta+E)^{2}}\left(1-\cos \left(2 k_{j} a_{1}\right)\right)\right\}
$$

The exponent is a finite quantity. Therefore, up to zeroth order, the level width is finite as $N \rightarrow \infty$. This is true as long as the height of barriers is finite.

The summation in the exponent of (23) can be approximated by an integral. It turns out that,

$$
\prod_{j=1}^{\infty}\left|t_{j}\right| \sim \exp \left\{-\alpha\left(\frac{V_{0}}{4}\right)^{2} \frac{1}{\Delta(J \Delta+E)}\right\}
$$


where $\alpha$ is a number of order 1 . We can see that, as $\Delta$ becomes very small ( but not smaller than $E / N)$, the level width shrinks to zero with a rate $\sim \exp (-1 / \Delta)$. In the $\Delta \rightarrow 0$ limit, (24) is no longer valid. In this case, $j \Delta+E \simeq E$, and the tunneling coefficient turns out to be $\simeq \exp \left(-\alpha\left(V_{0} / 4\right)^{2}\left(N / E^{2}\right)\right)$, which is finite as long as the system is finite.

We can also reproduce the leading term in the exponent of (19) for the case of the Kronig-Penny model in the limit that $a_{1} \rightarrow 0, V_{0} \rightarrow \infty$, and at the same time keep $a_{1} V_{0}$ a constant.

\section{Conclusion}

We have calculated the local density of states $\rho(x, E)$ for a 1D model using delta function potential barriers to simulate a periodic potential and a staircase potential to simulate an external electric field. The W-S ladders are revealed most clearly as peaks of the local density of states in 3D plots of $\rho(x, E)$. We have demonstrated that the ladder structures can be easily washed out in the total density of states.

We have also offered a new approach, based on the calculation of leakage current in a graded array of potential barriers, to evaluate the level width.[12] This complements the usual method based on the picture of Zener tunneling between Bloch bands.[13] It is shown that, when the system size $N$ is very large, the level width $\delta E \sim N^{(-\alpha / \Delta)}$ in the case of $\delta$ barriers. We choose $\delta$ potential barriers to simplify the derivation of the transfer matrices as well as transmission and reflection coefficients. The same approach can be applied to nonsingular potentials. We found that, when the potential barriers are rectangular, the reflection coefficient vanishes like $1 / x$ for large $x$, and that the energy level width will not shrink to zero as the size of the system becomes infinite. When the potential is smoother, the reflection coefficient vanishes even faster. The electron wave function can be localized

only in the case of a $\delta$ potential chain, but even then the localization is found to be only marginal; that is, it obeys a power law.

Some of the general properties of W-S ladders are summarized below, and these should also be valid in a real solid. Firstly, the number of sequencies presented in a finite system can be estimated from a tilted-band diagram. It can be controlled either by changing the 
size of the system, or, more conveniently, by changing the electric field. Secondly, among these sequences, there is one that is most pronounced. This is best illustrated in Fig. 3 (d). The states of the sequence that are closest to the staircase are sharper and more localized spatially. This can be explained by the theory in Sec.4. The states in this sequence have initial barriers which are difficult to tunnel through. The states in the other sequences are more remote from the origin, and therefore have higher energy with respect to the ground. This makes them easier to tunnel through. Thirdly, within the same sequence, the states in cell -1 are narrower than those in cell 0 , and those in cell 0 are narrower than those in cell 1 ...etc. This is because the state in cell -1 has to go through more barriers in order to tunnel out. A similar kind of asymmetry leads to the asymmetrical absorption spectra in the experiment that was done by Agulló-Rueda et al [9].

\section{Acknowledgment}

The authors wish to thank W.Kohn, L.Kleinman and P.Ao for many valuable discussions. This work is supported in part by the Texas Advanced Research Program, by a Precision Measurement grant from NIST, and by a fellowship on the Trull Centennial Professorship at the University of Texas at Austin.

\section{References}

1. G.H.Wannier, Phys. Rev. 117, 432 (1960).

2. J.Zak, Phys. Rev. Lett. 20, 1477 (1968) ; A.Rabinovitch Phys. Lett. 33A, 403 (1970)

; J.E.Avoron, J.Zak, A.Grossmann and L.Gunther, J.Math.Phys. 18, 918 (1977).

3. A.Rabinovitch and J,Zak, Phys. Rev. B4, 2358 (1971).

4. K.Hacker and G.Obermair Z. phys. 234, 1 (1970).

5. A.Rabinovitch and J.Zak Phys. Lett. 40A,189 (1972) ; J.N.Churchill and F.E.Holmstrom, Phys. Lett. 85A 453 (1981).

6. D.Emin and C.F.Hart, Phys. Rev. B36, 7353 (1987); L.Kleiman, Phys. Rev. B41, 3857 (1990) ; P.N.Argyres and S.Sfiat, Phys. Lett. 146A, 231 (1990) ; J.Zak, Phys. Rev. B43, 4519 (1991). 
7. S.Maekawa, Phys. Rev. Lett.24, 1175 (1970); R.Koss and L.Lambert, Phys. Rev. B5, 1479 (1972). J.R.Banavas and D.D.Coon, Phys. Rev. B17, 3744 (1978) ; F.Bentosela, V.Grecchi and F.Zironi, J.Phys. C: Solid State Phys. 15, 7119 (1982).

8. J.B.Krieger and G.J.Iafrate, Phys. Rev. B33, 5494 (1986).

9. To quote a few of them: J.Bleuse, G.Bastard and P.Voison, Phys. Rev. Lett. 60, 220 (1988) ; F.Agulló-Rueda, E.E.Mendez and J.M.Hong, Phys. Rev. B40, 1357 (1989) on optical absorption ; explained via exiton effect by M.M.Dignam and J.E.Sipe, Phys. Rev. Lett. 64, 1797 (1990). M.Nakayama, Phys. Rev. Lett. 65, 2720 (1990) on electroreflectance. M.K.Saker, Phys. Rev. B43, 4945 (1991) on photoconductivity spectra.

10. J.Zak, Phys. Lett. 76A, 287 (1980).

11. G.Blatter and D.A. Browne, Phys. Rev. B37, 3856 (1988) ; P.Ao Phys. Rev B41, 3998 (1990).

12. It is interesting to observe that similar phenomenon as W-S ladders can also be found if electron wave function is replaced by EM wave, see G.Monsivais and M.CastilloMussot, Phys. Rev. Lett. 64, 1433 (1990).

13. A.M.Berezhkovskii and A.A.Ovchinnikov, Sov. Phys. Solid State, 18, 1908 (1976). 


\section{Figure Captions}

Fig.1. The potential of the system described in equations $(1),(2)$. It corresponds to the case $N_{r}=N_{l}=5$.

Fig.2. The plot of $\rho(E)$ when electric field is zero, $\left(V_{0}, \Delta, N_{r}\right)=(0.2,0,40) . N_{l}$ in this and in all of the following figures are fixed to be 10 .

Fig.3 (a),(b). Local density of states $\rho(x, E)$ plotted as a function of $x$ and $E$. $\left(V_{0}, \Delta, N_{r}\right)=(0.2,0.2,40) 12$ cells are plotted $(-1 \sim 10)$. Range of energy covered is 3 $\Delta$, spatial resolution is $a / 5$, energy resolution is $\Delta / 100$. The curves will be smoother with higher resolution.

Fig.4 (a),(b),(c),(d). A series of plots of $\rho(E)$ for a system with $\left(V_{0}, \Delta\right)=(0.2,0.2)$, $N_{r}=40,60,100,160$ respectively. (a) is for the system plotted in Fig. 3. Also note the difference between (a) and Fig. 2.

Fig.5 (a),(b). Two plots of $\rho(E)$ for a system with $\left(V_{0}, \Delta\right)=(0.2,1), N_{r}=40,160$ respectively. The electric field of the systems in this figure is 5 times larger than those in Fig. 4.

Fig.6. Plots of the states at $E_{1}=0.339063 \Delta$, Fig.(a) ; and $E_{2}=0.6757468519 \Delta$, Fig.(b), in a system with $\left(V, \Delta, N_{r}\right)=(1,0.2,40)$.

Fig.7. A cross section of the peak in cell $3(x=3.5 a)$ in Fig. 6(b). The range of energy covered is extremely small. $E_{c}=0.67574685190 \Delta$, half width $\delta E=6 \times 10^{-11} \Delta$.

Fig.8. A cross section of the lowest peak in cell $0 .\left(V_{0}, \Delta, N_{r}\right)=(8,0.2,4) . E_{c}=$ $4.7607583641 \Delta, \delta E=6.4 \times 10^{-10} \Delta$. Notice that the strength of the $\delta$ function barriers is 8 time large. The number of cells on the positive $x$ axis is only 4 . The level lies only slightly below the level of an infinitely deep square well, which is $5 \Delta$.

Fig.9. (a) A path that makes its last return at point $j$. We call the bold-faced part a self energy loop of $j . \Sigma_{j}$ is the summation of every possible self energy loops of $j$. (b) A self energy loop with only two reflections. The associated tunneling amplitude is stated in (12). $\sigma_{j}^{(2)}$ is the summation of every possible self energy loops of this kind. 